\title{
High Annexin A5 expression promotes tumor progression and poor prognosis in renal cell carcinoma
}

\author{
JINGYUAN TANG $^{1,2^{*}}$, ZHIQIANG QIN $^{1 *}$, PENG HAN $^{1^{*}}$, WEI WANG $^{1}$, CHENGDI YANG $^{1}$, ZHEN XU $^{1}$, \\ RAN LI $^{1}$, BIANJIANG LIU ${ }^{1}$, CHAO QIN $^{1}$, ZENGJUN WANG ${ }^{1}$, MIN TANG $^{1}$ and WEI ZHANG ${ }^{1}$ \\ ${ }^{1}$ Department of Urology, The First Affiliated Hospital of Nanjing Medical University, Nanjing, Jiangsu 210009; \\ ${ }^{2}$ Department of Urology, Wuxi Second People's Hospital, Nanjing Medical University, Wuxi, Jiangsu 214002, P.R. China
}

Received November 28, 2016; Accepted March 20, 2017

DOI: $10.3892 /$ ijo.2017.3942

\begin{abstract}
Annexin A5 has been found to act as an oncogenic protein in a variety of cancers. However, its specific biological role and mechanism in renal cell cancer (RCC) remains unknown. Quantitative Real-time PCR and western blotting were used to evaluate the mRNA and protein expression level of Annexin A5 in human RCC cell lines and tissues. Immunohistochemistry was adopted to measure the Annexin A5 expression in 123 cases of RCC tissues. Survival analysis was performed to explore the association between Annexin A5 expression and the prognosis of RCC. The effect of Annexin A5 on RCC growth and metastasis was studied in vitro and in vivo. Annexin A5 was frequently highly expressed in both human RCC cells and tissues. High Annexin A5 expression was associated with higher clinical stage and histological grade. In addition, Annexin A5 might be used as a predictive factor for the prognosis of RCC. Further research suggested that upregulated Annexin A5 in RCC cells could significantly promote tumor cell proliferation, migration and invasion in vitro. Subcutaneous xenograft tumor model displayed that knockdown of Annexin A5 could impede tumorigenesis in vivo. Moreover, mechanism study exhibited that Annexin A5 could activate PI3K/Akt/mTOR signaling pathway, promote epithelial-mesenchymal transition (EMT) and the expression of MMP2 and MMP9. Annexin A5 may be a potential prognostic biomarker in RCC and promotes proliferation, migration and invasion of RCC cells via activating PI3K/Akt/mTOR signaling pathway and regulating EMT process and MMP expression.
\end{abstract}

\section{Introduction}

Renal cell carcinoma ( $\mathrm{RCC}$ ) is the most common type of kidney cancer with approximately 62,700 new RCC cases

Correspondence to: Dr Min Tang or Dr Wei Zhang, Department of Urology, The First Affiliated Hospital of Nanjing Medical University, 300 Guangzhou Road, Nanjing, Jiangsu 210029, P.R. China

E-mail: mintang@njmu.edu.cn

E-mail: zhangwei_urology@163.com

*Contributed equally

Key words: Annexin A5, renal cell cancer, prognosis, proliferation diagnosed and 14,240 new mortalities in the United States in 2016, accounting for approximately 2-3\% of all adult malignancies (1). Clear cell renal cell carcinoma (ccRCC), as the most common histological subtype of RCC, represents approximately $85 \%$ of all primary renal neoplasms (2). Due to lack of early warning signs and effective treatments for patients with advanced disease, approximately 20-40\% patients were found metastasis at the time of diagnosis and the 5-year survival rate of RCC is approximately 55\% $(1,3,4)$. However, the accurate mechanism of the RCC progression remains unclear. Therefore, there is an urgent need to improve the understanding of tumor biology in RCC and identify more effective and highly selective potential therapeutic targets for RCC treatment.

Annexins, as predominantly cytosolic soluble proteins, are classified into five families, including vertebrates, invertebrates, fungi and some groups of unicellular eukaryotes, plants and protists. Annexins can reversibly bind to negatively charged phospholipids in a $\mathrm{Ca}^{2+}$ regulated manner (5). Twelve Annexins common to vertebrates are known as Annexins A1-A11 and A13 $(5,6)$. The non-glycosylated phospholipid binding protein, Annexin A5 is composed of 319 amino acid residues with a molecular mass of $\sim 35.7 \mathrm{kDa}$ (7-9). It is likely that Annexin A5 has a very short unphosphorylated N-terminus compared with other Annexins, contributing to a series of functions, such as cell proliferation and invasion $(6,10)$, signal transduction $(11,12)$, and anticoagulation (13). Previous studies have reported that Annexin A5 promoted tumorigenesis and progression in a variety of cancers, including hepatocarcinoma, colorectal cancer and breast cancer $(14,15)$. However, the possible relationship between Annexin A5 and RCC is not clear. Therefore, we analyzed the expression level of Annexin A5 in RCC tissue samples compared with normal renal tissue samples and explored its potential biomedical functions on RCC cell invasion to confirm whether Annexin A5 is a new molecular biomarker of RCC.

\section{Materials and methods}

Patients and RCC samples. All primary RCC and pericarcinous tissues were obtained from 123 patients with appropriate informed consent at the Department of Urology of the First Affiliated Hospital of Nanjing Medical University from 
February 2008 to August 2011. The follow-up deadline was January 2016. The specimens were assessed by immunohistochemistry and the diagnosis was verified by histopathological examination. The study was approved by the Institutional Research Ethics Committee of the First Affiliated Hospital of Nanjing Medical University.

Quantitative real-time PCR ( $q R T-P C R)$. Total RNA was extracted from cultured cell lines and clinical samples using TRIzol reagent (Invitrogen, Carlsbad, CA, USA) and cDNA was synthesized using Primescript RT Reagent (Takara, Otsu, Japan) according to the manufacturer's instructions. The qRT-PCR was performed by using StepOne Plus Real-time PCR system (Applied Biosystems, Foster City, CA, USA) with SYBR $^{\circledR}$ Premix Ex Taq ${ }^{\mathrm{TM}}$ Reagent (Takara). The following primers were used for qRT-PCR: Annexin A5, forward: 5'-AGCGGGCTGATGCAGAAAC-3', reverse: 5'-ACTTCG GGATGTCAACAGAGT-3'; $\beta$-actin, forward: 5'-CCTGGC ACCCAGCACAAT-3', reverse: 5'-GCTGATCCACATCTG CTGGAA-3'. Data analysis was performed with ABI Step One Software version 2.1 and the relative mRNA level was calculated using $2^{-\Delta \Delta \mathrm{Ct}}$ method.

Western blotting. Cells or frozen tissues were lysed in cell lysis buffer for $30 \mathrm{~min}$ on ice and centrifuged at $14,000 \mathrm{x}$ g at $4{ }^{\circ} \mathrm{C}$ for $15 \mathrm{~min}$. The total protein concentration was calculated by the BCA Protein Assay kit (Pierce, Rockford, IL, USA). Proteins were separated by $10 \%$ SDS-PAGE gel and transferred onto a polyvinylidene difluoride membrane (Millipore, Billerica, MA, USA). Western blot analysis followed a standard procedure. The primary antibody Annexin A5 was obtained from Santa Cruz Biotechnology, Inc. (Santa Cruz, CA, USA). $\mathrm{N}$-cadherin, vimentin, $\beta$-catenin, MMP2, MMP9, PI3K, phospho-PI3K, AKT, phospho-AKT, mTOR, phospho-mTOR were obtained from Cell Signaling Technology, Danvers, MA, USA. The anti-mouse and anti-rabbit secondary antibodies were also from Cell Signaling Technology.

Cell lines and reagents. The human renal cancer cell lines (Caki-1, Caki-2, ACHN, 769P) and normal epithelial cells of renal tubule (HK2) were obtained from the American Type Culture Collection (ATCC, Manassas, VA, USA). The cells were cultured in McCoy's 5A or DMEM media supplemented with $10 \%$ fetal bovine serum (Gibco, Carlsbad, CA, USA) and $1 \%$ penicillin/streptomycin (Invitrogen) at $5 \% \mathrm{CO}_{2}$ and $37^{\circ} \mathrm{C}$ incubator. LY294002, a phosphatidylinositol 3-kinase (PI3K) inhibitor, was obtained from Selleck Chemical (Houston, TX, USA) (no. S1105).

Transfection. Lentivirus packaging cells were transfected with LV3-pGLV-h1-GFP-puro vector (GenePharma, Shanghai, China) containing either the Annexin A5 knockdown (shA5-1 and shA5-2) or Annexin A5 overexpression (A5) and a negative control sequence (NC), respectively. Lentiviral transduction was performed in Caki-1 and Caki-2 cell lines. Pools of stable transductants were generated by selection using puromycin $(4 \mu \mathrm{g} / \mathrm{ml})$ for 2 weeks.

Cell proliferation assay. A Cell Counting Kit-8 assay (Dojindo Laboratories, Kumamoto, Japan) was used to estimate the proliferation potential. Cells were seeded in 96-well plates with 3000 cells/well. CCK-8 reagents were added into wells after cells grew for 1, 2, 3, and 4 days, respectively, and the absorbance was measured at $450 \mathrm{~nm}$ using a micro-plate reader at $2 \mathrm{~h}$ after CCK-8 addition.

Colony formation assay. Cells were seeded into 6-well plates (600 cells/well) and cultured in media containing 10\% FBS for 2 weeks. Then the colonies were fixed with paraform and stained with $0.1 \%$ crystal violet. The colonies were counted and each group were repeated three times.

Transwell cell migration and invasion assay. Cell migration or invasion assay were performed using a 24-well Transwell chamber (Costar, Corning, NY, USA) with or without Matrigel (Invitrogen). Cells $\left(2 \times 10^{4}\right)$ were suspended in serum-free medium and seeded into the upper chambers which were inserted in the 24 -well plate. Medium containing $10 \%$ FBS was added to the lower chamber. Cells were incubated at $37^{\circ} \mathrm{C}$ for $48 \mathrm{~h}$ and then cells on the surface of the upper chambers that did not migrate through the pores were removed with a cotton swab. Cells which migrated to the bottom surface of the chamber were stained with $0.1 \%$ crystal violet. Numbers of migratory and invasive cells were counted in five randomly selected fields and the presented data represent three individual experiments.

Xenograft studies. Mouse studies were approved by the Animal Research Ethics Committee of Nanjing Medical University. The 5-week-old female nude mice were randomly divided into two groups consisting of five mice each. The stable cells $\left(7 \times 10^{6}\right)$ shA5-Caki-1 and the control cells (NC-Caki-1) were suspended in $150 \mu \mathrm{l}$ PBS and injected subcutaneously into the flank of each mouse. Tumor size was calculated (length $\mathrm{x}$ width ${ }^{2} \mathrm{x} 0.52$ ) once a week. After 6 weeks, tumors were removed, weighed, fixed, and embedded for immunohistochemical staining.

Immunohistochemistry. Immunohistochemistry was performed on tissue microarray to evaluate Annexin A5 protein expression. Tissue microarray was incubated with a primary antibody against Annexin A5 at $4{ }^{\circ} \mathrm{C}$ overnight and incubated with HRP conjugated secondary antibody followed by DAB staining. Immunohistochemistry staining was assessed by two experienced pathologists. To evaluate the expression of Annexin A5 in RCC tissues, a semi-quantitative scoring system (0-3) was used based on the staining intensity of the tumor tissue: 0 , negative; 1 , weak positive; 2 , moderate positive; 3 , strong positive. Annexin A5 high expresion refers to scores 2-3 and Annexin A5 low expresion refers to scores 0-1. For assessing the association of Annexin A5 expression with clinicopathological characteristics of the RCC patients, following parameters were included: age $(\leq 60$ and $>60)$, gender, tumor size ( $\leq 4$ and $>4)$, Histology (clear cell carcinoma and others), histological stage (grades I, II, III, and IV) and tumor stage (TNM stages I, II, III, and IV).

Statistical analysis. Statistical analyses were performed with SPSS 22.0 software. All the data were presented as the mean \pm SD from three independent experiments. Student's 

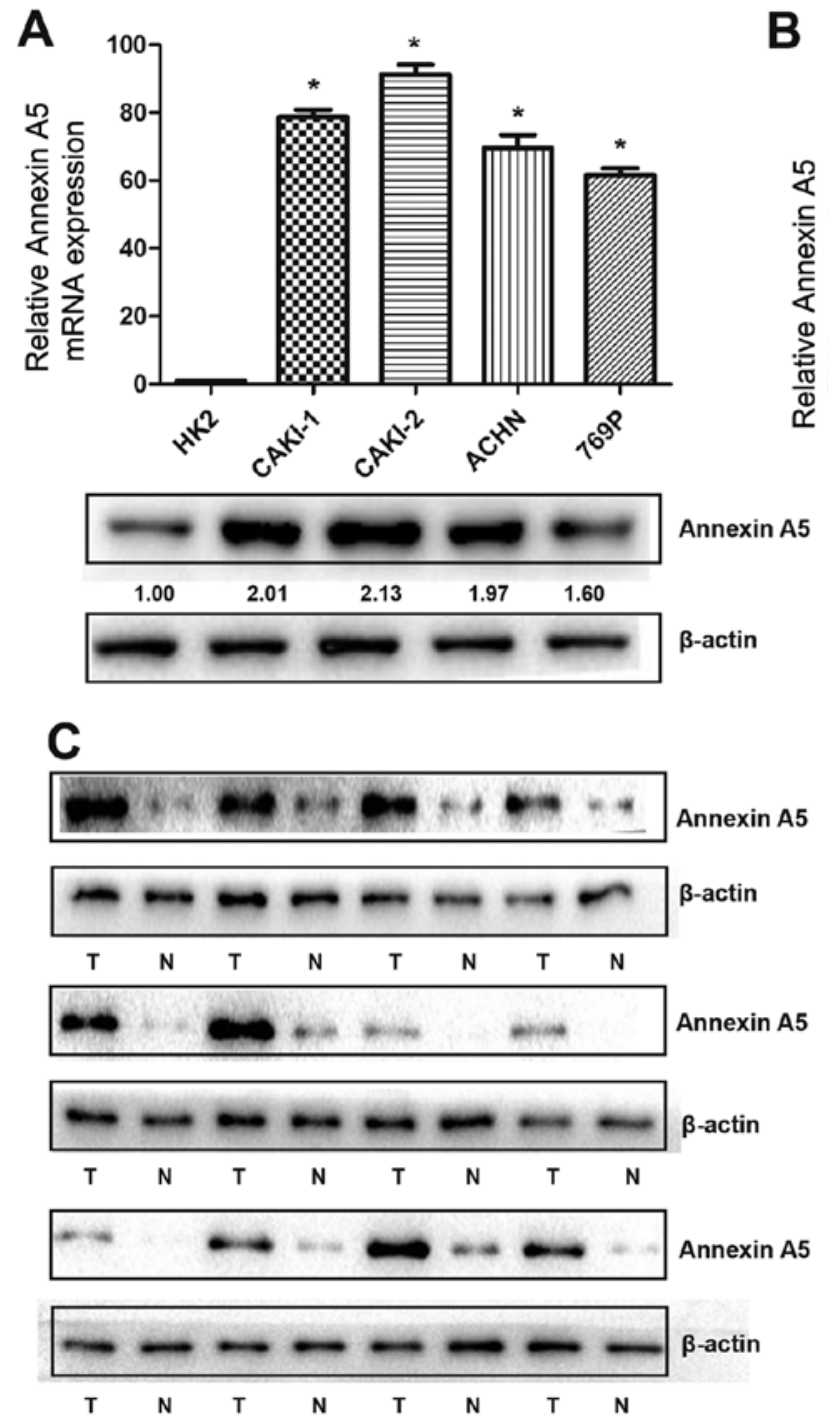

B

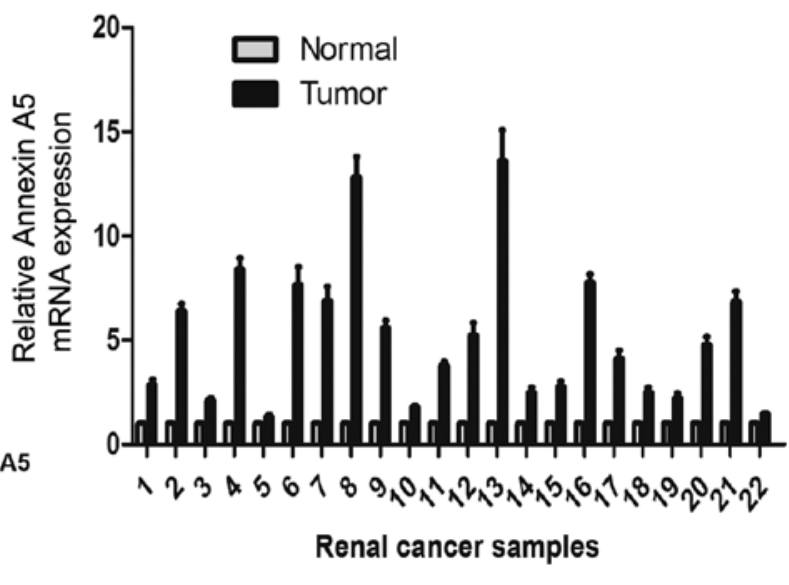

D

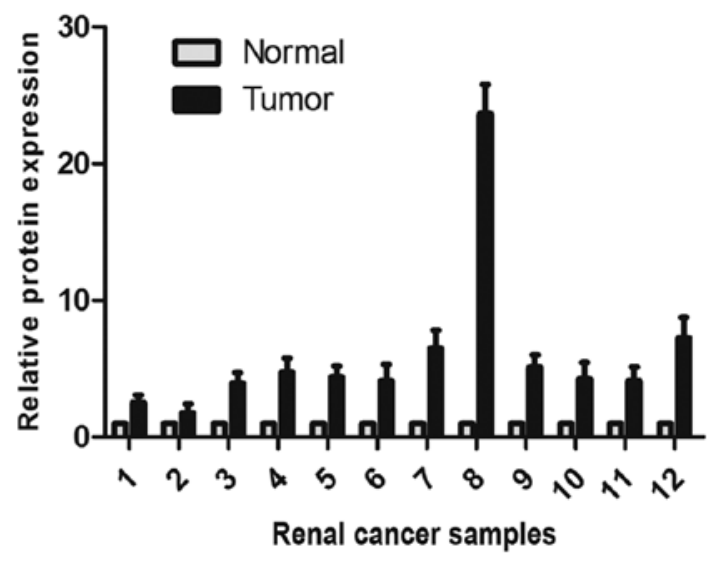

Figure 1. The expression of Annexin A5 in RCC cell lines and tissues. (A) qRT-PCR and western blot analysis of Annexin A5 expression in RCC cell lines and normal epithelium cell of renal tubule HK2. The fold change of Annexin A5 is shown below each lane. The intensity of the bands was determined using densitometric analysis. (B) Annexin A5 mRNA expression in 22 pairs of RCC tissues and matched adjacent non-cancerous tissues. (C and D) Annexin A5 protein expression in 22 pairs of RCC tissues and adjacent tissues. Twelve representative cases are shown. Data are the mean \pm SD from three independent experiments, ${ }^{*} \mathrm{P}<0.05$.

t-test and the Chi-square test were used to analyze the differences between groups and survival curves were drawn by the Kaplan-Meier method. $\mathrm{P}<0.05$ was considered to indicate a statistical significant difference.

\section{Results}

Annexin A5 is highly expressed in RCC tissues and cell lines. To explore the protein and mRNA expression of Annexin A5 in RCC cell lines, western blot and qRT-PCR were performed in four human RCC cell lines (Caki-1, Caki-2, ACHN and 769P) and one normal epithelial cell of renal tubule (HK2). As shown in Fig. 1A, Annexin A5 mRNA was upregulated by 61.2 - to 92.4 -fold in all RCC cell lines compared to HK2 and western blot analysis showed similar results. To assess whether Annexin A5 was also highly expressed in RCC tissues, 22 pairs of RCC tissues and matched adjacent non-cancerous tissue were selected for qRT-PCR and western blot, the results suggested that Annexin A5 expression was markedly upregulated in both protein and mRNA level compared to the adjacent tissue (Fig. 1B-D).

Annexin A5 expression is correlated with clinical stage, histological grade and overall survival in RCC patients. To validate the association between Annexin A5 expression and clinicopathologic features, 123 cases of RCC were evaluated by IHC. Patients were divided into a low expression group $(n=56)$ and a high expression group $(n=67)$ according to the Annexin A5 expression. As shown in Table I, although Annexin A5 expression and age $(\mathrm{P}=0.628)$, gender $(\mathrm{P}=0.576)$, tumor size $(\mathrm{P}=0.192)$ or tumor histology $(\mathrm{P}=0.637)$ did not significantly correlate, Annexin A5 exhibited a signifcantly positive correlation with histological grade $(\mathrm{P}=0.007)$ and TNM stage $(\mathrm{P}=0.013)$. High expression group was correlated with higher histological grade and TNM stage compared with the low expression group. As shown in Fig. 2, RCC patients with high Annexin A5 expression had a significantly shorter overall survival time than those with low Annexin A5 expression $(\mathrm{P}=0.031)$. These data suggested 
Table I. Association of Annexin A5 expression with clinicopathological characteristics of the renal cancer patients.

\begin{tabular}{|c|c|c|c|c|}
\hline \multirow[b]{2}{*}{ Parameters } & \multirow[b]{2}{*}{$\begin{array}{c}\text { No. of } \\
\text { cases }(\%)\end{array}$} & \multicolumn{2}{|c|}{$\begin{array}{c}\text { Annexin A5 } \\
\text { expression }\end{array}$} & \multirow[b]{2}{*}{ P-value } \\
\hline & & $\begin{array}{c}\text { Low } \\
(\%)\end{array}$ & $\begin{array}{c}\text { High } \\
(\%)\end{array}$ & \\
\hline Age (years) & & & & 0.628 \\
\hline$\leq 60$ & $74(60.2)$ & $35(47.3)$ & $39(52.7)$ & \\
\hline$>60$ & $49(39.8)$ & $21(42.9)$ & $28(57.1)$ & \\
\hline Gender & & & & 0.576 \\
\hline Male & $78(63.4)$ & $37(47.4)$ & $41(52.6)$ & \\
\hline Female & $45(36.6)$ & $19(42.2)$ & $26(57.8)$ & \\
\hline Tumor size $(\mathrm{cm})$ & & & & 0.192 \\
\hline$\leq 4$ & $58(47.2)$ & $30(51.7)$ & $28(48.3)$ & \\
\hline$>4$ & $65(52.8)$ & $26(40.0)$ & $39(60.0)$ & \\
\hline Histology & & & & 0.637 \\
\hline Clear cell carcinoma & $115(93.5)$ & $53(46.1)$ & $62(53.9)$ & \\
\hline Others & $8(6.5)$ & $3(37.5)$ & $5(62.5)$ & \\
\hline Histological grade & & & & 0.007 \\
\hline I-II & $102(82.9)$ & $52(51.0)$ & $50(49.0)$ & \\
\hline III-IV & $21(17.1)$ & $4(19.0)$ & $17(81.0)$ & \\
\hline TNM stage & & & & 0.013 \\
\hline I & $95(77.2)$ & $49(51.6)$ & $46(48.4)$ & \\
\hline II-IV & $28(22.8)$ & $7(25.0)$ & $21(75.0)$ & \\
\hline
\end{tabular}

that the upregulated expression of Annexin A5 might have an important role during RCC progression.

Annexin A5 promotes cell proliferation in vitro. To further investigate the functions of Annexin A5 in RCC cell lines, we transfected Caki-1 and Caki-2 cells with lentivirus to silence or overexpress the expression of Annexin A5. The silenced cells were named as shA5-1 and shA5-2 and the overexpressed cell lines were called A5, while the matched control cells were named as NC. The expression levels were confirmed by western blotting (Fig. 3A and E) and qRT-PCR (Fig. 3B and F, $\mathrm{P}<0.01$ ).

The growth curve analysis demonstrated that the downregulation of Annexin A5 in Caki-1 and Caki-2 cells significantly inhibited cell growth compared with the control cells (Fig. 3C and D, P<0.05). Whereas, upregulated Annexin A5 expression enhanced cell growth markedly in Caki-1 and Caki-2 cells (Fig. $3 \mathrm{G}$ and $\mathrm{H}, \mathrm{P}<0.05$ ). In addition, a colony formation assay was also performed to further investigate the effect of Annexin A5 on the proliferation. The results indicated that Annexin A5 knockdown contributed significantly to decrease cell colony formation efficiency, whereas inducing Annexin A5 expression markedly enhanced the ability of Caki-1 and Caki-2 cells to form colonies (Fig. 4A and B, $\mathrm{P}<0.05$ ).

To further study the mechanism by which Annexin A5 overexpression or knockdown affected proliferation, we

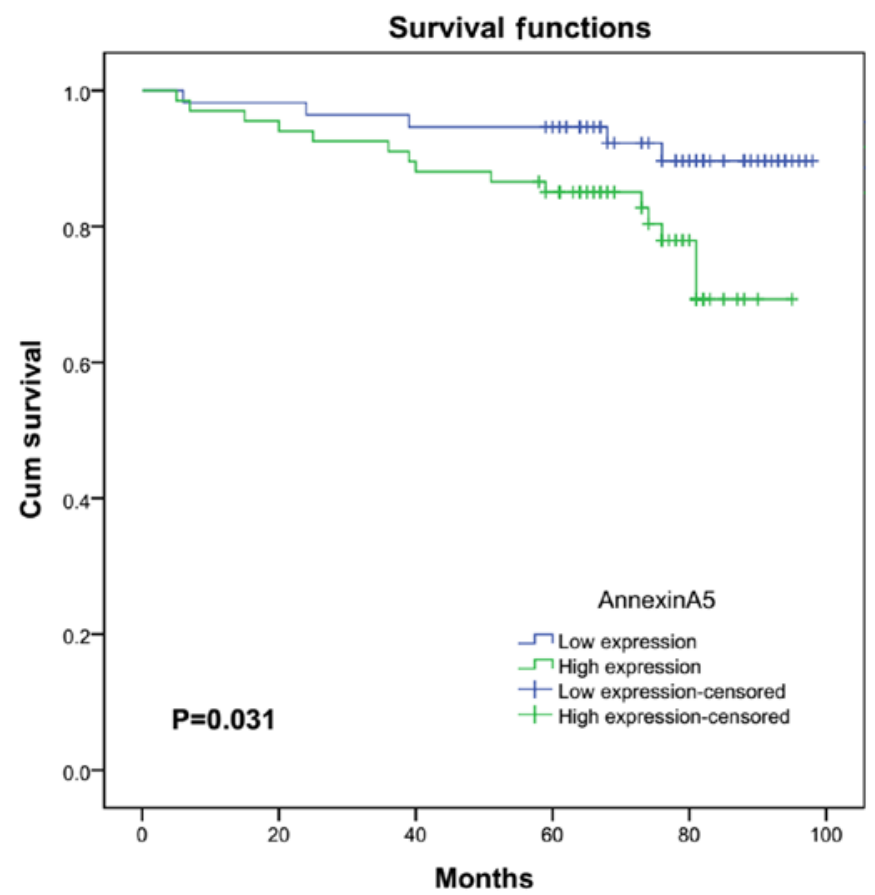

Figure 2. Kaplan-Meier survival curves of patients with RCC based on Annexin A5 expression. Patients in the high expression group had a significantly more unfavorable prognosis than those in low expression group $(\mathrm{P}=0.031, \log$-rank test $)$.

investigated the effects of Annexin A5 overexpression or knockdown on the PI3K/AKT/mTOR pathway. Western blot analysis suggested that the levels of phospho-PI3K, phospho-AKT and phospho-mTOR decreased in the Annexin A5 knockdown cells, while increased in the Annexin A5 over-expressed cells. However, the total levels of PI3K, AKT and mTOR had no obvious change in Annexin A5 overexpression or knockdown cells (Fig. 4C and D, P<0.05).

To further confirm our hypothesis, we used PI3K inhibitor (LY294002) at a dose of $20 \mu \mathrm{M}$ to observe the role of PI3K/AKT/mTOR pathway in Annexin A5-regulated cell proliferation. The dose of LY294002 selected was the dose of $\mathrm{IC}_{50}$ to Caki-1 cells. After treated with LY294002 for $48 \mathrm{~h}$, Caki-1 and Caki-2 with Annexin A5 overexpression (A5) and control cells (NC) were used for CCK8 analysis. As shown in Fig. 4E, Annexin A5 overexpression increased the cell proliferation while LY294002 reversed the cell growth promoting trend by Annexin A5. Overall, these results suggested that Annexin A5 positively regulated cell proliferation in RCC and might be mediated by the phosphorylation of $\mathrm{PI} 3 \mathrm{~K} / \mathrm{AKT} / \mathrm{mTOR}$ pathway.

Annexin A5 promotes cell migratory and invasive potential. To assess the potential role of Annexin A5 in regulating the migration and invasion ability of RCC cells, Transwell migration and invasion assays were performed in Caki-1 and Caki-2 cells with Annexin A5 overexpression or knockdown. Transwell migration assays revealed that Annexin A5 knockdown reduced the migration capability of Caki-1 and Caki-2 cells and overexpressing Annexin A5 signifcantly increased the ability of migration. The Transwell invasion assays showed similar results (Fig. 5A-D, $\mathrm{P}<0.01$ ). 
A
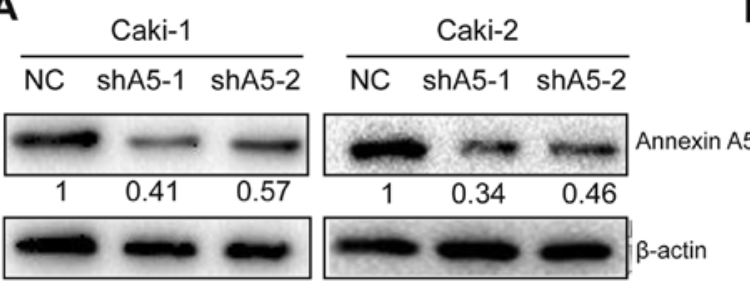

B

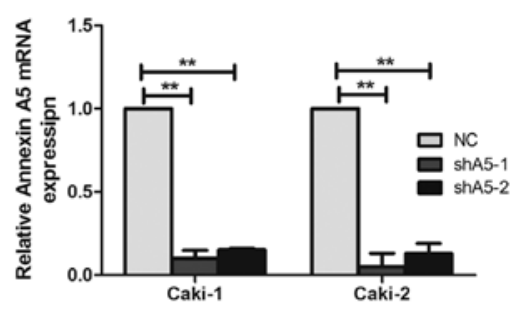

C

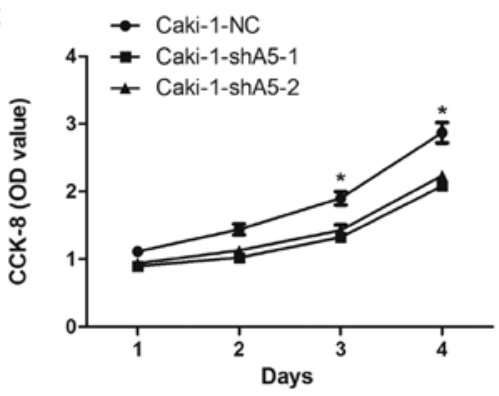

$\mathbf{E}$

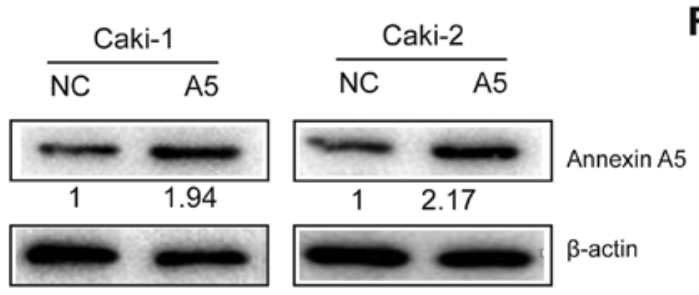

G

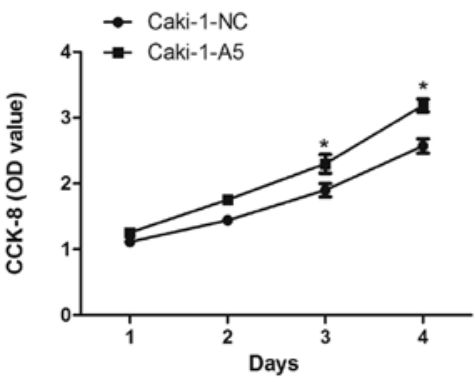

D

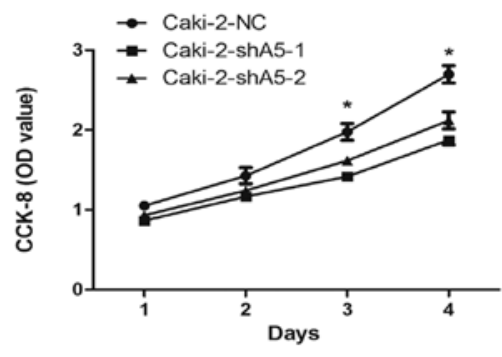

$\mathbf{F}$

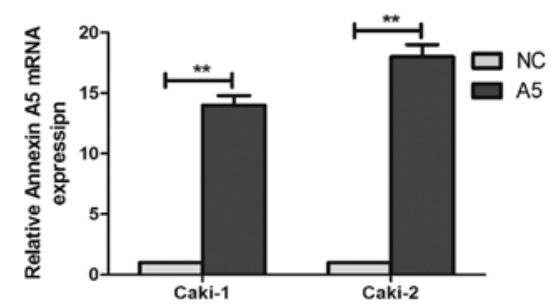

H

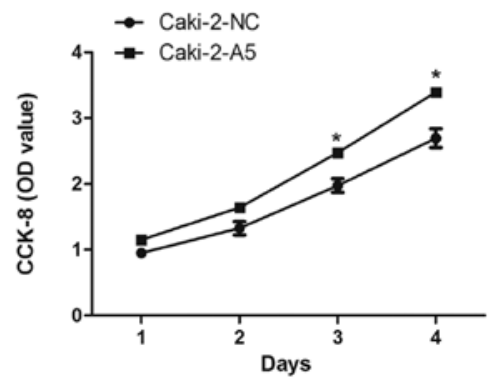

Figure 3. Effect of Annexin A5 on proliferation and growth of RCC cell lines Caki-1 and Caki-2. (A) Western blotting and (B) qRT-PCR were used to verify the efficiency of Annexin A5 knockdown. shA5-1 and shA5-2 indicates Annexin A5 knockdown; NC indicates cells transfected with an empty vector; the fold change of Annexin A5 is shown below each lane. (C and D) Growth curve analysis showing the cell growth of Caki-1 and Caki-2 cells with Annexin A5 knockdown. (E) Western blotting and (F) qRT-PCR were used to verify the efficiency of Annexin A5 overexpression (named as A5). (G and H) Growth curve analysis showing the cell growth of Caki-1 and Caki-2 cells with Annexin A5 overexpression. Data represent the mean \pm SD from three independent experiments, ${ }^{*} \mathrm{P}<0.05,{ }^{* *} \mathrm{P}<0.01$.

To further explore the mechanism by which Annexin A5 affects cell migration and invasion, western blotting was performed to determine the expression levels of $\mathrm{N}$-cadherin, vimentin, $\beta$-catenin, MMP2 and MMP9, which are related to regulating the cell adhesion and metastasis. The results demonstrated that the expression of these proteins was significantly downregulated in Annexin A5 knockdown cells while they were upregulated in Annexin A5 overexpressing cells (Fig. 5E and F). These data suggested that Annexin A5 could promote migration and invasion potential in RCC cells and the underlying mechanism might be the upregulation of adhesion and metastasis-related molecules.

Knockdown of Annexin A5 impedes tumorigenesis in vivo. To study the effect of Annexin A5 expression on tumor growth in vivo, we constructed subcutaneous xenograft tumor model using Caki-1 cells with Annexin A5 knockdown and control cells in the female nude mice. As shown in Fig. 6A and B, tumors from the Annexin A5 knockdown group (shA5) grew markedly slower than those from control group (NC). The mean tumor weight of shA5 group was lower compared to the NC group (Fig. 6C) and Ki-67, a proliferation marker of tumor, was markedly decreased in tumors from shA5 group (Fig. 6D and E). Collectively, these results showed that Annexin A5 knockdown could significantly impede tumor growth in vivo.

\section{Discussion}

RCC remains a major clinical challenge due to its poor long-term prognosis. Various oncogenes and suppressor genes 
A
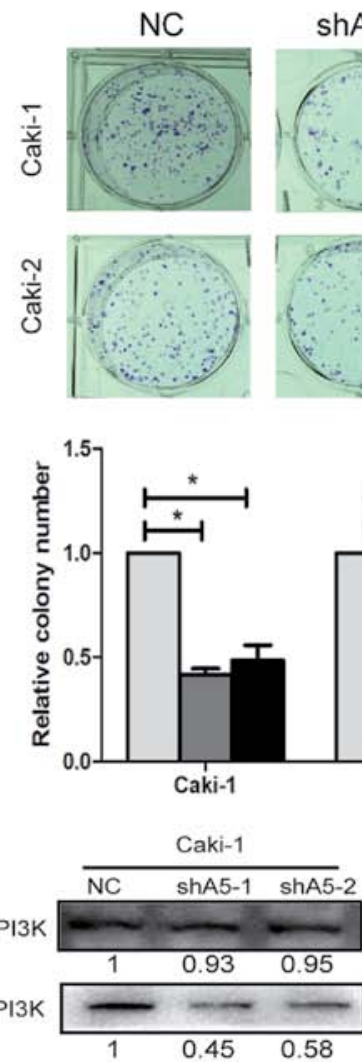

AKT
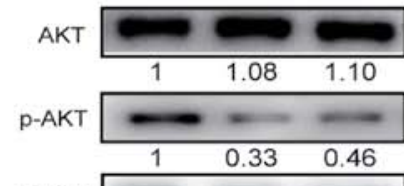

mTOR

p-mTOR

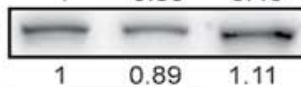

-

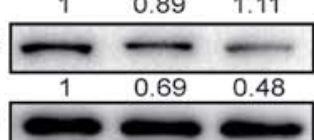

E

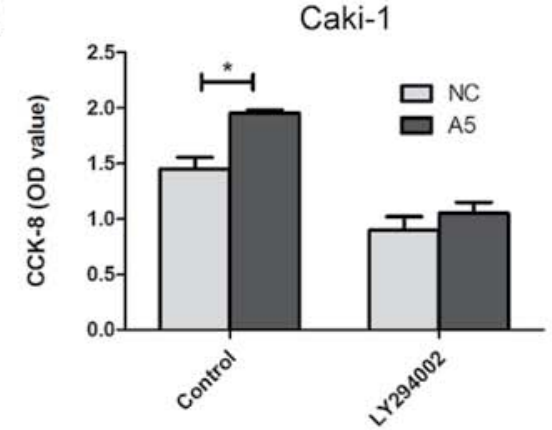

shA5-2
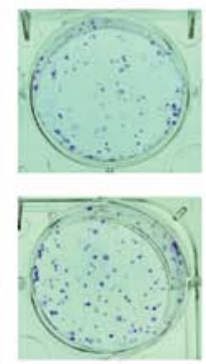

$\square$ NC

$\square$ shA5-1

- shA5-2
B
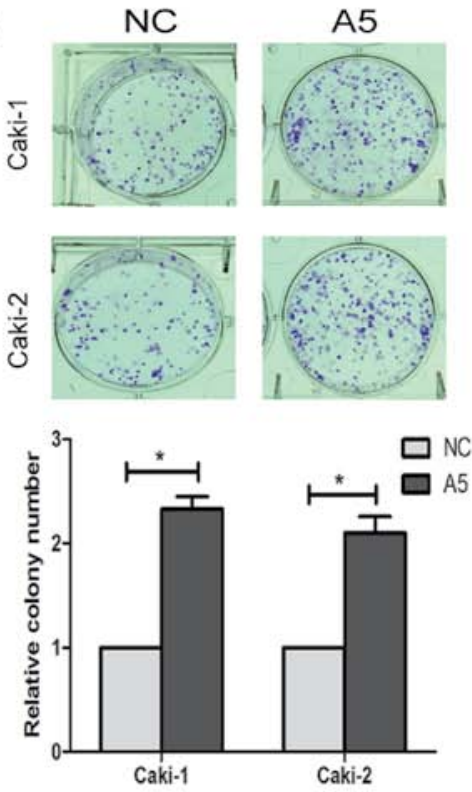

D

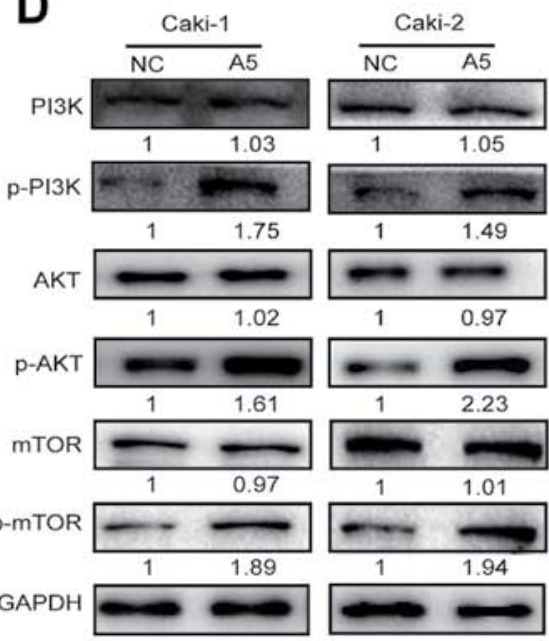

Caki-2

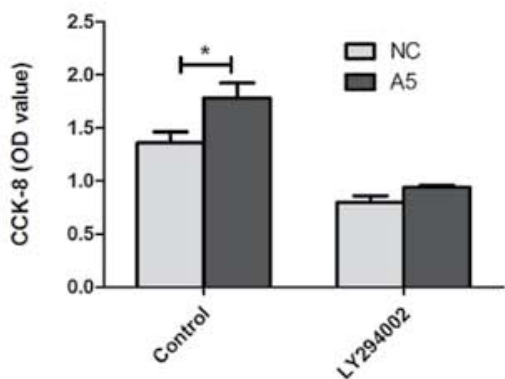

Figure 4. Colony-forming growth assay and molecular mechanisms responsible for Annexin A5-induced proliferation. (A and B) The efficiency of cell colony formation in Caki-1 and Caki-2 cells with Annexin A5 knockdown or overexpression. (C and D) PI3K, phospho-PI3K, AKT, phospho-AKT, mTOR, phospho-mTOR protein expression levels were analyzed by western blotting in Caki-1 and Caki-2 cells with Annexin A5 knockdown or overexpression. The fold change of protein expression is shown below each lane. (E) The proliferation ability of Caki-1 and Caki-2 with Annexin A5 overexpression (A5) and control cells (NC) was examined by CCK8 after treated with LY294002 for $48 \mathrm{~h}$. Data represent the mean \pm SD from three independent experiments, ${ }^{*} \mathrm{P}<0.05$, ${ }^{* *} \mathrm{P}<0.01$

are involved in the tumorigenesis of RCC (15). Therefore, it is urgent to find appropriate RCC biomarkers which are essential to tumor progression. Recent studies have suggested that Annexin A5, a calcium-dependent phospholipid-binding protein, could promote tumorigenesis and progression in certain types of cancers $(14,15,17)$. This study focused on the biological functions of Annexin A5 and its potential clinical value in RCC.

Among the RCC cell lines analyzed, Annexin A5 was found to be highly expressed in RCC cells compared to HK2. Consistent with this, Annexin A5 was frequently upregulated in RCC tissues and acted as a predictor of clinical stage, 
A
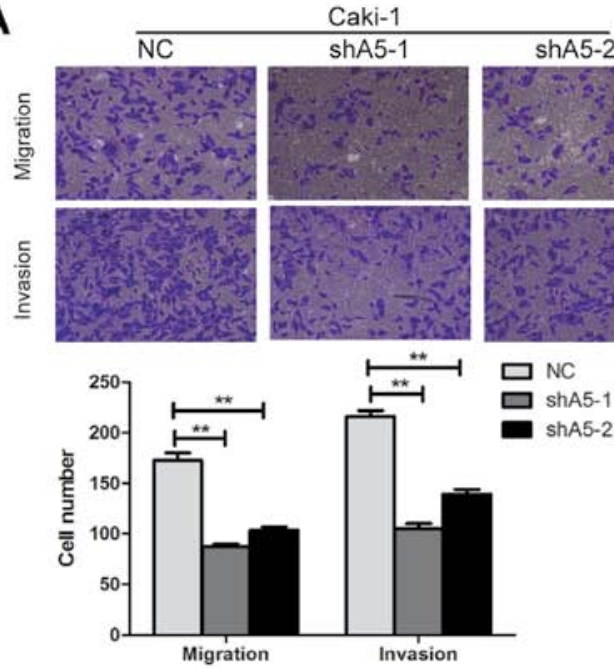

C
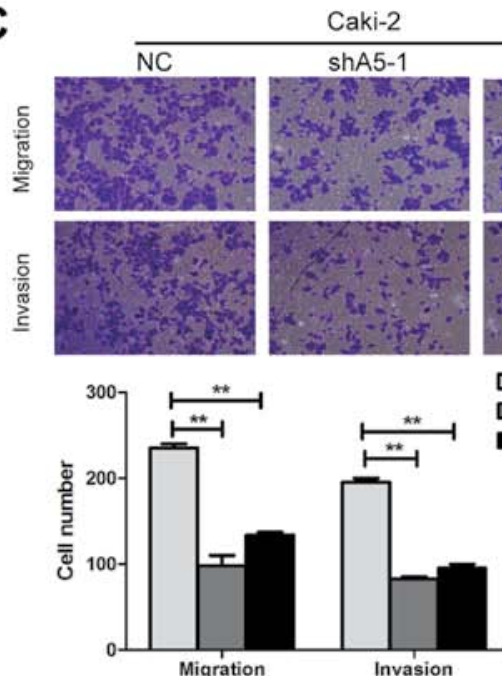

E

Caki-1

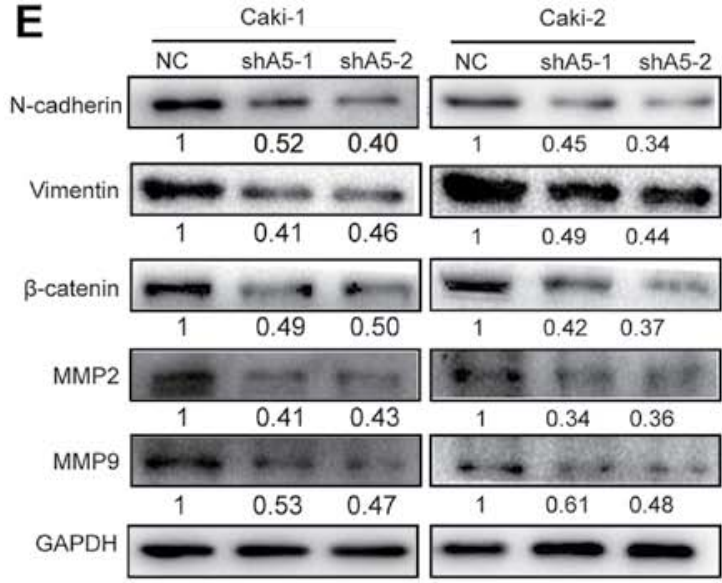

B
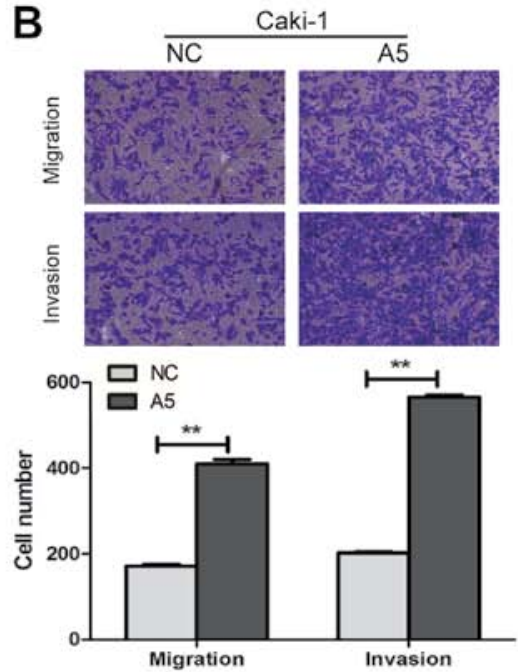

D
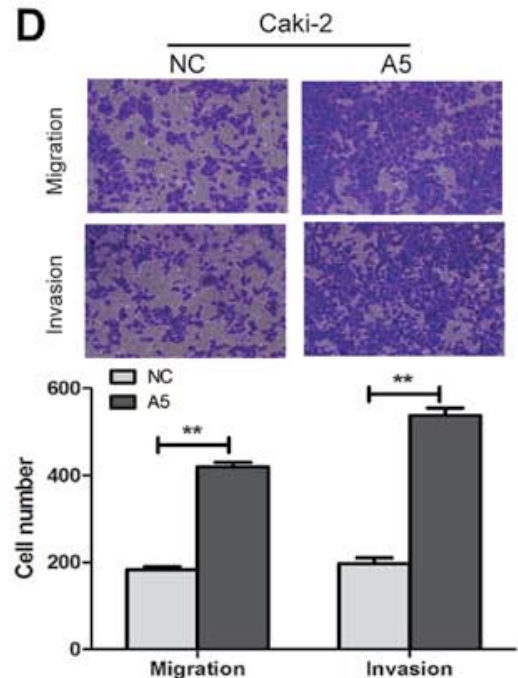

$\mathrm{F}$

N-cadherin

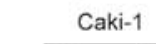

Caki-2
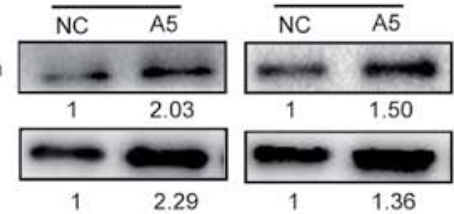

MMP2
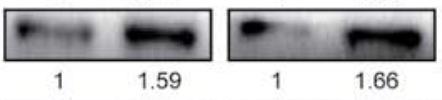

MMP9

GAPDH
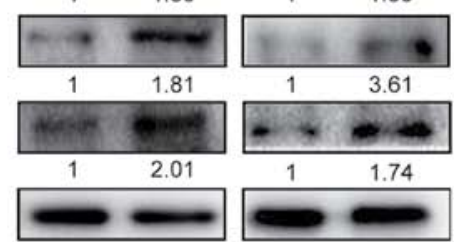

Figure 5. Annexin A5 promotes cell migratory and invasive potential and its underlying mechanism. (A-D) Transwell migration assay and Matrigel invasion assay in Caki-1 and Caki-2 cells with Annexin A5 knockdown or overexpression. (E and F) N-cadherin, vimentin, $\beta$-catenin, MMP2, MMP9 protein expression levels were analyzed by western blotting in Caki-1 and Caki-2 cells with Annexin A5 knockdown or overexpression. The fold change of protein expression is shown below each lane. Data represent the mean $\pm \mathrm{SD}$ from three independent experiments, ${ }^{*} \mathrm{P}<0.05,{ }^{* *} \mathrm{P}<0.01$.

histological grade and overall survival in RCC. Therefore, Annexin A5 might be used as a clinical marker for RCC, although more pathological data are required to confirm this conclusion. Moreover, two RCC cell lines Caki-1 and Caki-2 were used as cell models to demonstrate the potential role of Annexin A5 in RCC cells. In both cell lines, knockdown of Annexin A5 markedly reduced cell growth rate and colony formation efficiency, while overexpression of Annexin A5 signifcantly accelerated cell proliferation. Similarly, knockdown of Annexin A5 increased, whereas overexpression of Annexin A5 decreased the migratory and invasive potential in RCC cells. In addition, xenograft studies showed Annexin A5 downregulated cells formed smaller tumors compared to the control cells. These data suggested that Annexin A5 played as 

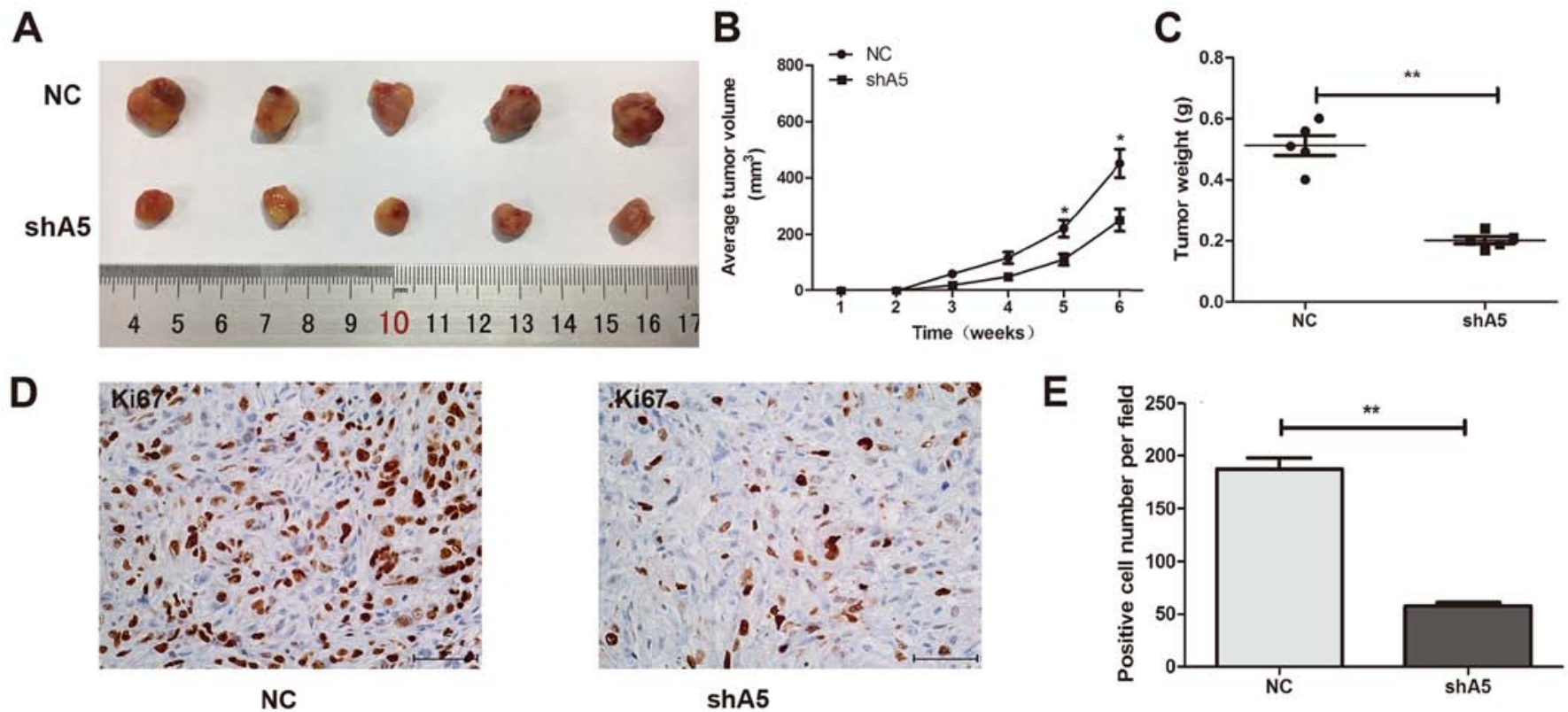

Figure 6. Knockdown of Annexin A5 impedes tumorigenesis in vivo. (A) Representative pictures of tumor in Caki-1-NC and Caki-1-shA5 cell-transplanted mice. (B and C) The tumor volume and weight were measured at the indicated weeks after mice were transplanted. (D) Cell proliferation was evaluated by $\mathrm{Ki}-67$ immunohistochemistry in xenografs. (E) Statistical analysis of Ki-67 positive cells from the panel. Data represent the mean \pm SD from three independent experiments, ${ }^{*} \mathrm{P}<0.05,{ }^{* *} \mathrm{P}<0.01$.

a tumor promoter in RCC. To the best of our knowledge, this is the first study to explore the relationship between Annexin A5 and RCC tumorigenesis and progression.

It has been reported that Annexin A5 was upregulated in various cancers including hepatocarcinoma (16), breast cancer (17), cervical cancer (18), colorectal adenocarcinoma (19) and glioma (20). In this study, Annexin A5 was also highly expressed in RCC which was in accord with the previous studies. However, Annexin A5 was negatively related with tumorigenesis in thyroid cancer and diffuse large B-cell lymphoma $(21,22)$. Therefore, Annexin A5 might play a dual role in cancer depending on tissue specificity.

Uncontrolled proliferation and inappropriate cell survival are one of the characteristics in cancer and these processes are commonly regulated by PI3K/Akt/mTOR pathway (23). The $\mathrm{PI} 3 \mathrm{~K} / \mathrm{Akt} / \mathrm{mTOR}$ signaling pathway is activated by several tyrosine kinase receptors and plays an important role in the regulation of many aspects of cell function including metabolism, proliferation, protein synthesis and survival (24). It has been reported that the abnormal expression of Annexin A5 may lead to aberrant activation of PKC and cellular signal transduction, which may contribute to tumorigenesis (14). In glioblastoma multiforme (GBM), Annexin A5 promoted GBM progression and chemoresistance to temozolomide through a PI3K-dependent mechanism (25). Our study demonstrated that overexpression of Annexin A5 activated the PI3K/Akt/mTOR signaling pathway which might be involved in RCC cell proliferation. Moreover, PI3K inhibitor (LY294002) could reverse the cell growth promoting trend by Annexin A5.

Migration and invasion are the major events in the metastasis of cancer (26). The EMT refers to the conversion of epithelial cells to mesenchymal cells, which is crucial in the progress of tumor metastasis (27). EMT is characterized by a loss of E-cadherin and an increase in non-epithelial cadherins including $\mathrm{N}$-cadherin and vimentin (28). MMPs belong to a family of zinc-dependent endopeptidases which can degrade the extracellular matrix (29). Especially, MMPs have remarkable effects on tumor invasion and metastasis (30). To explore the underlying mechanism by which Annexin A5 contributes to cell migration and invasion of RCC, we investigated the expression of EMT-related proteins and MMPs. Among the tested cell lines, $\mathrm{N}$-cadherin, vimentin and $\beta$-catenin were significantly upregulated in Annexin A5 overexpression group and Annexin A5 markedly increased MMP2 and MMP9 expression. Therefore, we propose that Annexin A5 may promote migration and invasion of RCC by regulating EMT and extracellular matrix degradation. However, the role of these metastasis-related makers in Annexin A5-induced progression of RCC should be further studied in vivo in subsequent studies.

In addition, Annexin A5 also enhanced chemoresistance in gastric cancer and nasopharyngeal carcinoma which might be associated with the multidrug resistance protein (MRP) (14). A study showed that the protein expression of MRP and Annexin A5 were concurrently elevated in drug-resistance of gastric cancer cells SGC-7901/DDP compared to SGC-7901. Downregulation of Annexin A5 in SGC-7901/DDP cells decreased the expression of MRP and increase the sensitivity to cisplatin, paclitaxel and 5-Fu (31). Future studies are required to explore the role of Annexin A5 in the chemosensitivity of RCC. Moreover, Annexin A1, A2, A4, and A5 played a vital role in breast cancer, pancreatic cancer, and laryngeal carcinoma, alone and/or synergistically (32). It would be of interest to investigate whether other Annexin family members play a role, alone or coupled with Annexin A5 in RCC.

In conclusion, our study suggested that Annexin A5 was frequently highly expressed in RCC and might be used as a novel prognostic indicator for RCC. In vitro and in vivo 
experiments validated the promotion function of Annexin A5 in RCC proliferation and metastasis via activating the $\mathrm{PI} 3 \mathrm{~K} / \mathrm{Akt} / \mathrm{mTOR}$ signaling pathway and regulating EMT process and MMPs expression. Annexin A5 may become a novel therapeutic target and prognosis factor for the future treatment of RCC.

\section{Acknowledgements}

This work was supported by the National Natural Science Funding of China (nos. 81600514, 81370781, 81670608).

\section{References}

1. Siegel RL, Miller KD and Jemal A: Cancer statistics, 2016. CA Cancer J Clin 66: 7-30, 2016

2. Yu W, Wang Y, Jiang Y, Zhang W and Li Y: Genetic analysis and clinicopathological features of ALK-rearranged renal cell carcinoma in a large series of resected Chinese renal cell carcinoma patients and literature review. Histopathology: Feb 15, 2017 (Epub ahead of print). doi: 10.1111/his.13185.

3. Golovastova MO, Korolev DO, Tsoy LV, Varshavsky VA, Xu WH, Vinarov AZ, Zernii EY, Philippov PP and Zamyatnin AA Jr: Biomarkers of renal tumors: The current state and clinical perspectives. Curr Urol Rep 18: 3, 2017.

4. Choueiri TK and Motzer RJ: Systemic therapy for metastatic renal-cell carcinoma. N Engl J Med 376: 354-366, 2017.

5. Mirsaeidi M, Gidfar S, Vu A and Schraufnagel D: Annexins family: Insights into their functions and potential role in pathogenesis of sarcoidosis. J Transl Med 14: 89, 2016.

6. Laohavisit A and Davies JM: Annexins. New Phytol 189: 40-53, 2011.

7. Bouter A, Carmeille R, Gounou C, Bouvet F, Degrelle SA, Evain-Brion D and Brisson AR: Review: Annexin-A5 and cell membrane repair. Placenta 36 (Suppl 1): S43-S49, 2015.

8. Lauritzen SP, Boye TL and Nylandsted J: Annexins are instrumental for efficient plasma membrane repair in cancer cells Semin Cell Dev Biol 45: 32-38, 2015.

9. Fatimathas L and Moss SE: Annexins as disease modifiers. Histol Histopathol 25: 527-532, 2010.

10. Mussunoor S and Murray GI: The role of annexins in tumour development and progression. J Pathol 216: 131-140, 2008.

11. Gerke V, Creutz CE and Moss SE: Annexins: Linking $\mathrm{Ca}^{2+}$ signalling to membrane dynamics. Nat Rev Mol Cell Biol 6 : 449-461, 2005.

12. Rescher U and Gerke V: Annexins - unique membrane binding proteins with diverse functions. J Cell Sci 117: 2631-2639, 2004.

13. Hayes MJ and Moss SE: Annexins and disease. Biochem Biophys Res Commun 322: 1166-1170, 2004.

14. Peng B, Guo C, Guan H, Liu S and Sun MZ: Annexin A5 as a potential marker in tumors. Clin Chim Acta 427: 42-48, 2014.

15. Guo W, Xue J, Shi J, Li N, Shao Y, Yu X, Shen F, Wu M, Liu S and Cheng S: Proteomics analysis of distinct portal vein tumor thrombi in hepatocellular carcinoma patients. J Proteome Res 9: 4170-4175, 2010

16. Cojocaru E, Lozneanu L, Giuşcă SE, Căruntu ID and Danciu M Renal carcinogenesis - insights into signaling pathways. Rom J Morphol Embryol 56: 15-19, 2015.
17. Hong M, Park N and Chun YJ: Role of annexin a5 on mitochondria-dependent apoptosis induced by tetramethoxystilbene in human breast cancer cells. Biomol Ther (Seoul) 22: 519-524, 2014.

18. Bae SM, Lee CH, Cho YL, Nam KH, Kim YW, Kim CK, Han BD, Lee YJ, Chun HJ and Ahn WS: Two-dimensional gel analysis of protein expression profile in squamous cervical cancer patients. Gynecol Oncol 99: 26-35, 2005.

19. Xue G, Hao LQ, Ding FX, Mei Q, Huang JJ, Fu CG, Yan HL and Sun SH: Expression of Annexin A5 is associated with higher tumor stage and poor prognosis in colorectal adenocarcinomas. J Clin Gastroenterol 43: 831-837, 2009.

20. Rajcevic U, Petersen K, Knol JC, Loos M, Bougnaud S, Klychnikov O, Li KW, Pham TV, Wang J, Miletic H, et al: iTRAQ-based proteomics profiling reveals increased metabolic activity and cellular cross-talk in angiogenic compared with invasive glioblastoma phenotype. Mol Cell Proteomics 8: 2595-2612, 2009.

21. Sofiadis A, Becker S, Hellman U, Hultin-Rosenberg L, Dinets A, Hulchiy M, Zedenius J, Wallin G, Foukakis T, Höög A, et al: Proteomic profiling of follicular and papillary thyroid tumors. Eur J Endocrinol 166: 657-667, 2012.

22. Wang J, Zhang Y, Liu X, Ma J, Liu P, Hu C and Zhang G: Annexin A5 inhibits diffuse large B-cell lymphoma cell invasion and chemoresistance through phosphatidylinositol 3-kinase signaling. Oncol Rep 32: 2557-2563, 2014.

23. Yu JS and Cui W: Proliferation, survival and metabolism: The role of PI3K/AKT/mTOR signalling in pluripotency and cell fate determination. Development 143: 3050-3060, 2016

24. Li X, Wu C, Chen N, Gu H, Yen A, Cao L, Wang E and Wang L: $\mathrm{PI} 3 \mathrm{~K} / \mathrm{Akt} / \mathrm{mTOR}$ signaling pathway and targeted therapy for glioblastoma. Oncotarget 7: 33440-33450, 2016.

25. Wu L, Yang L, Xiong Y, Guo H, Shen X, Cheng Z, Zhang Y, Gao Z and Zhu X: Annexin A5 promotes invasion and chemoresistance to temozolomide in glioblastoma multiforme cells. Tumour Biol 35: 12327-12337, 2014.

26. Jin K, Li T, van Dam H, Zhou F and Zhang L: Molecular insights into tumour metastasis: Tracing the dominant events. J Pathol: Dec 30, 2016 (Epub ahead of print). doi: 10.1002/path.4871.

27. Thiery JP and Sleeman JP: Complex networks orchestrate epithelial-mesenchymal transitions. Nat Rev Mol Cell Biol 7: 131-142, 2006.

28. Itoigawa $\mathrm{Y}$, Harada N, Harada S, Katsura Y, Makino F, Ito J, Nurwidya F, Kato M, Takahashi F, Atsuta R, et al: TWEAK enhances TGF- $\beta$-induced epithelial-mesenchymal transition in human bronchial epithelial cells. Respir Res 16: 48, 2015.

29. Kapral M, Wawszczyk J, Jurzak M, Dymitruk D and Weglarz L: Evaluation of the expression of metalloproteinases 2 and 9 and their tissue inhibitors in colon cancer cells treated with phytic acid. Acta Pol Pharm 67: 625-629, 2010.

30. Wang Q, Yu W, Huang T, Zhu Y and Huang C: RUNX2 promotes hepatocellular carcinoma cell migration and invasion by upregulating MMP9 expression. Oncol Rep 36: 2777-2784, 2016.

31. Wu X, Tang Y, Huang W and Wu Y: Identification of proteins interacting with multidrug resistance protein in gastric cancer. World J Gastroenterol 19: 3568-3673, 2011.

32. Deng S, Wang J, Hou L, Li J, Chen G, Jing B, Zhang X and Yang Z: Annexin A1, A2, A4 and A5 play important roles in breast cancer, pancreatic cancer and laryngeal carcinoma, alone and/or synergistically. Oncol Lett 5: 107-112, 2013. 